\title{
Turn-on Fluorescent Dopamine Sensing Based on in Situ Formation of Visible Light Emitting Polydopamine Nanoparticles
}

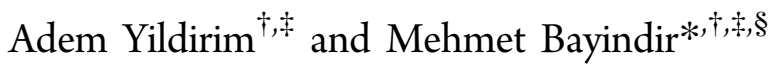 \\ ${ }^{\dagger}$ UNAM-National Nanotechnology Research Center, Bilkent University, 06800 Ankara, Turkey \\ ${ }^{*}$ Institute of Materials Science and Nanotechnology, Bilkent University, 06800 Ankara, Turkey \\ ${ }^{\S}$ Department of Physics, Bilkent University, 06800 Ankara, Turkey
}

\section{Supporting Information}

ABSTRACT: Dopamine is the principle biomarker for diseases such as schizophrenia, Huntington's, and Parkinson's, and the need is urgent for rapid and sensitive detection methods for diagnosis and monitoring of such diseases. In this Article, we report a turn-on fluorescent method for rapid dopamine sensing which is based on monitoring the intrinsic fluorescence of in situ synthesized polydopamine nanoparticles. The assay uses only a common base and an acid, $\mathrm{NaOH}$ and

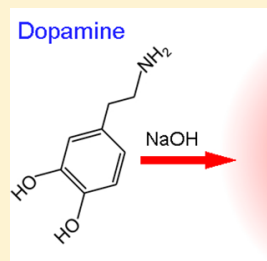
Fluorescent PDA nanoparticles

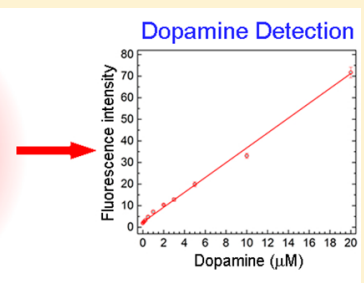
$\mathrm{HCl}$ to initiate and stop the polymerization reaction, respectively, which makes the assay extremely simple and low cost. First, we studied the in situ optical properties of polydopamine nanoparticles, for the first time, which formed under different alkaline conditions in order to determine optimum experimental parameters. Then, under optimized conditions we demonstrated high sensitivity (40 nM) and excellent selectivity of the assay. With its good analytical figures of merit, the described method is very promising for detection of dopamine related diseases.

$\mathrm{D}$ opamine (DA), a catecholamine neurotransmitter, regulates many biological processes in central nervous, hormonal, and cardiovascular systems. ${ }^{1,2}$ Abnormal DA concentrations in biological fluids (e.g., urine, blood plasma, and extracellular fluid of the central nervous system) can be indicator of several diseases such as schizophrenia and Huntington's and Parkinson's diseases. ${ }^{3-5}$ In this regard, sensitive and selective measurement of DA levels is important for diagnosis of these diseases and monitoring of patients. ${ }^{6}$ Common DA detection methods utilize electrochemical analysis, $^{7-9}$ chromatography coupled with spectroscopy ${ }^{10,11}$ (e.g., high-pressure liquid chromatography (HPLC)-fluorescence and gas chromatography/mass spectrometry (GC/MS)) and fluorescent ${ }^{12-16}$ or colorimetric probes ${ }^{1,17}$ (e.g., organic dyes, quantum dots, and gold nanoparticles). These methods, however, have some limitations. For instance, interference from uric acid (UA) and ascorbic acid (AA) largely limits selectivity of electrochemical methods. Chromatographic methods on the other hand, are time-consuming, labor intensive, and expensive with complicated procedures. Similarly, synthesis of fluorescent or colorimetric probes for DA detection involves complicated and time-consuming procedures.

A straightforward, cost-effective and rapid alternative for DA detection is measuring the optical absorption of oxidation product of dopamine under alkaline conditions. ${ }^{18-22}$ These assays use only a common base (e.g., $\mathrm{NaOH}$ ) or other oxidants (e.g., enzymes) as a reagent, and DA concentration is determined by simply measuring the optical absorption of the resulting brownish oxidation product. Unfortunately, the method demonstrates a poor sensitivity around a few micromolar. In these oxidation studies, the product is assumed as a quinone derivative of dopamine. ${ }^{18}$ However, recent studies demonstrated that under alkaline conditions the quinone product is unstable and rapidly polymerized by covalent attachment and aggregation. ${ }^{23-25}$ The resulting polymer is commonly referred to as polydopamine (PDA), which is structurally very similar to the natural eumelanin polymers. ${ }^{26}$ Although, the fluorescence property of eumelanins is known for more than 40 years and there is ongoing research to understand the interesting optical properties of these materials; ${ }^{27-29}$ the fluorescence of PDA is largely unexplored. To our knowledge, only very recently Zhang et al. ${ }^{30}$ reported that purified PDA nanoparticles are fluorescent and can be used for cellular imaging.

Here, we explored the intrinsic emission properties of PDA nanoparticles which were synthesized by oxidizing DA under different conditions and demonstrated that it can be used as turn-on type fluorescent reporter of DA existence. The introduced method is based on monitoring the visible light emission of in situ synthesized PDA nanoparticles, and it is capable to detect very low DA concentrations (detection limit is $40 \mathrm{nM}$ ). Note that the reached detection limit is $20-750$-fold better than the methods that measure the absorption of oxidization solution of DA (see the Supporting Information,

Received: February 27, 2014

Accepted: May 6, 2014

Published: May 6, 2014 
Table S1). Initially, we studied the fluorescence property of PDA synthesized under different basic conditions. We observed that the emission spectrum of PDA is very dependent on time, base type, and its concentration. Then, under optimized conditions, we performed DA sensing experiments. Finally we showed that the method is very selective to DA, and no interference from AA or UA is observed.

\section{MATERIALS AND METHODS}

Materials. Dopamine, ascorbic acid, glucose, urea, sucrose, aspartic acid, alanine, glycine, lysine, and hydrochloric acid (37\%) were purchased from Sigma-Aldrich (U.S); sodium hydroxide was purchased from Merck (Germany). All chemicals were used as purchased.

Oxidization of Dopamine. A volume of $1.8 \mathrm{~mL}$ of freshly prepared dopamine $(100 \mu \mathrm{M})$ solution in phosphate buffered saline (PBS) (2 mM, pH 7.4) was oxidized by dropwise addition of $200 \mu \mathrm{L}$ of Tris base (final concentration in the solution is $10 \mathrm{mM}$ ) or $\mathrm{NaOH}$ (final concentration in the solutions are $0.5 \mathrm{mM}, 10 \mathrm{mM}$, and $20 \mathrm{mM}$ ) stock solutions. Time dependent evaluation of UV-vis or fluorescence spectra of the solutions were in situ recorded using UV-vis absorption (Cary 5000, Varian) and fluorescence (Eclipse, Varian) spectrophotometers, respectively.

Characterization of Polydopamine Nanoparticles. A volume of $5.4 \mathrm{~mL}$ of dopamine solutions $(100 \mu \mathrm{M})$ in PBS was polymerized using $0.6 \mathrm{~mL}$ of $\mathrm{NaOH}$ (final concentration in the solution is $20 \mathrm{mM}$ ) for $3 \mathrm{~h}$. Then the particles were purified using membrane dialysis. A volume of $2 \mu \mathrm{L}$ of purified particles dripped on a transmission electron microscopy (TEM) grid, and morphology of the particles were investigated using a TEM (Tecnai G2 F30, FEI). For dynamic light scattering (DLS) measurements, $100 \mu \mathrm{M}$ of dopamine was polymerized in PBS for $3 \mathrm{~h}$. Then particles were filtered with a $1 \mu \mathrm{m}$ syringe filter, and their size distribution was measured with Zetasizer Nanoseries (Malvern Instruments).

Stopping the Oxidization Reaction. In order to stop the polymerization reaction, excess acid can be added to the polymerization solution. For instance, $2 \mathrm{~mL}$ of dopamine oxidization solution in which the final concentrations of dopamine and $\mathrm{NaOH}$ are $100 \mu \mathrm{M}$ and $20 \mathrm{mM}$, respectively, is stopped using $42 \mu \mathrm{L}$ of $1 \mathrm{M} \mathrm{HCl}$.

Sensitivity and Selectivity Experiments. Freshly prepared dopamine stock solutions were diluted in PBS to give final volume of $1.8 \mathrm{~mL}$ and concentrations ranging from 0.1 to $20 \mu \mathrm{M}$. Polymerization reactions were initiated using $200 \mu \mathrm{L}$ of $\mathrm{NaOH}$ (final concentration is $20 \mathrm{mM}$ ). After $30 \mathrm{~min}$, reactions were stopped using $42 \mu \mathrm{L}$ of $1 \mathrm{M} \mathrm{HCl}$ and solutions were further incubated for $10 \mathrm{~min}$. Then, fluorescence spectra of solutions were recorded (excitation wavelength is $370 \mathrm{~nm}$ ). For selectivity experiments, concentration of all interfering chemicals (ascorbic acid, uric acid, urea, glucose, sucrose, aspartic acid, lysine, alanine, and glycine) was $0.1 \mathrm{mM}$ and other parameters were the same with the sensitivity experiments. All experiments were performed in triplicate.

Absorption Based Assay. The samples were prepared using the same experimental parameters with the fluorescence assay. Absorption of DA oxidization solutions (DA concentrations were between 0.5 and $20 \mu \mathrm{M}$ ) at $360 \mathrm{~nm}$ were recorded using a UV-vis absorption (Cary 100, Varian) spectrophotometer.

\section{RESULTS AND DISCUSSION}

Under alkaline conditions, DA is spontaneously oxidized to its quinone derivative and then autopolymerized to form PDA (Scheme 1). ${ }^{23,24}$ Polymerization of DA solution $(1 \mathrm{mM})$ in

Scheme 1. Schematic Representation of Fluorescent PDA Nanoparticle Formation

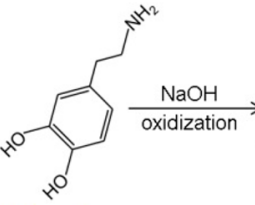

Dopamine

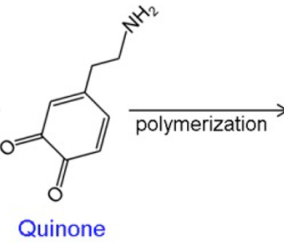

Quinone

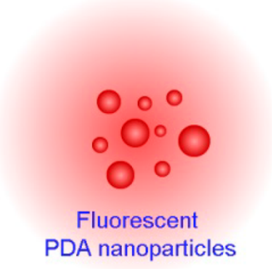

PBS ( $\mathrm{pH}$ 7.4) was in situ investigated using UV-vis spectroscopy for $3 \mathrm{~h}$ (see the Supporting Information, Figure $\mathrm{S} 1$ ). After $\mathrm{NaOH}$ addition (final concentration is $20 \mathrm{mM}$ ), a peak around $350 \mathrm{~nm}$ appeared which indicates the quinone formation ${ }^{24}$ and its intensity gradually increased for $3 \mathrm{~h}$. The broad peak around $470 \mathrm{~nm}$ shows the intramolecular cyclization of the quinone derivative ${ }^{24}$ which is an intermediate product in the polymerization of DA (see the Supporting Information, inset of Figure S1). ${ }^{23}$ After $10 \mathrm{~min}$, this broad peak disappeared and absorption at all wavelengths between 400 and $700 \mathrm{~nm}$ increased gradually, which shows the formation of $\mathrm{PDA}^{24}$ In order to characterize the PDA nanoparticles formed after $3 \mathrm{~h}$ of oxidization reaction, we polymerized $100 \mu \mathrm{M}$ DA using $20 \mathrm{mM} \mathrm{NaOH}$. Particle size distribution of PDA nanoparticles is given in Figure 1, which

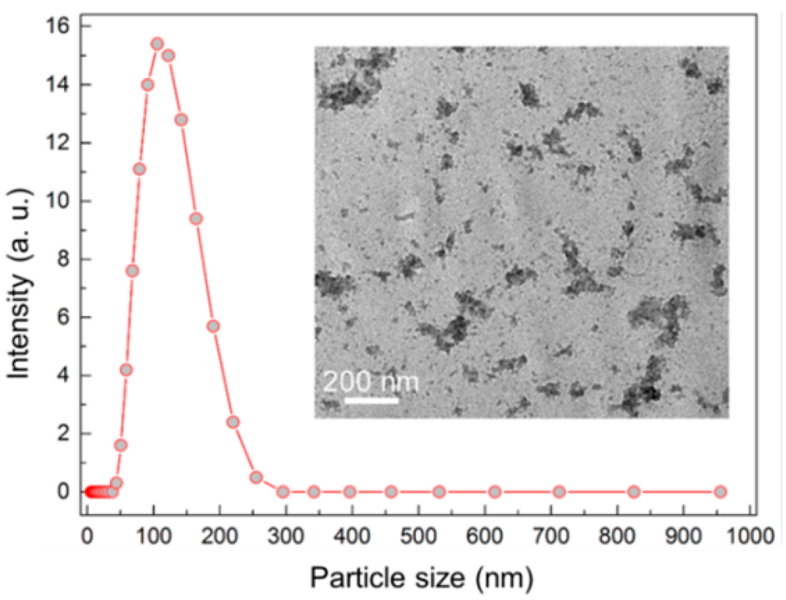

Figure 1. Particle size distribution of PDA nanoparticles. The graph shows the particle size distribution of PDA nanoparticles which were prepared after oxidization of $100 \mu \mathrm{M}$ DA solution for $3 \mathrm{~h}$ in the presence of $20 \mathrm{mM} \mathrm{NaOH}$. Inset shows the TEM images of the purified DA oxidization solution showing the polydisperse and irregularly shaped PDA nanoparticles.

shows the broad size distribution of particles with diameters ranging from a few nanometers to hundreds of nanometers. Figure 1 inset shows the TEM image of $3 \mathrm{~h}$ oxidized PDA particles. PDA nanoparticles were irregular in shape and have broad particle size distribution which is in accordance with DLS results. 
The resulting PDA nanoparticles are intrinsically fluorescent (will be discussed in detail below). Initially, we explored the effect of different oxidation conditions on the fluorescence of oxidation product of DA (i.e., PDA nanoparticles) in order to determine optimum experimental conditions for DA sensing. We compared two bases that are commonly applied in DA oxidization; $^{18,23}$ Tris base and $\mathrm{NaOH}$ at the same $\mathrm{pH}$ and molarity. We measured the in situ fluorescence of $100 \mu \mathrm{M}$ DA which is oxidized under different basic conditions for $1 \mathrm{~h}$ and compared the fluorescence intensities at $510 \mathrm{~nm}$ at different time intervals. The results are presented in Figure 2 and Figure

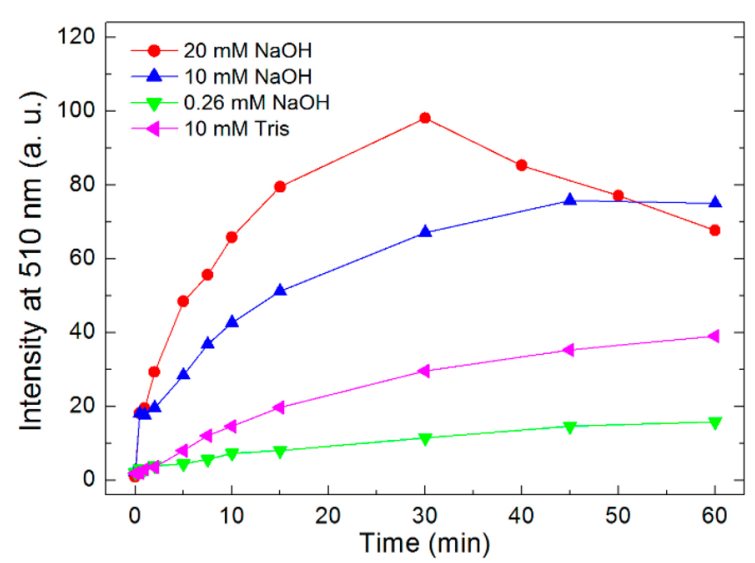

Figure 2. Time dependent fluorescence intensity at $510 \mathrm{~nm}$ of $100 \mu \mathrm{M}$ DA solutions, which were oxidized at different basic conditions.

S2 (Supporting Information). At the same $\mathrm{pH}$ value (9.6), Tris is more effective than $\mathrm{NaOH}$ in terms of generating fluorescent PDA nanoparticles. On the other hand, at the same molarity $(10 \mathrm{mM}), \mathrm{NaOH}$ is almost 2 times more effective than Tris base. Also, we explored fluorescence of the polymerization solution at different $\mathrm{NaOH}$ concentrations, and we selected 20 $\mathrm{mM}$ as optimal for the sensing experiments. Therefore, we used $20 \mathrm{mM} \mathrm{NaOH}$ to oxidize DA in the rest of the study.

The fluorescence spectra of synthesized PDA nanoparticles after $3 \mathrm{~h}$ of oxidization, which were prepared using $100 \mu \mathrm{M} \mathrm{DA}$, is given in Figure 3a. We observed that fluorescence behavior of the resulting PDA nanoparticles is very similar to eumelanin polymers in which the fluorescence spectrum is excitation wavelength dependent and can be tuned in the whole visible spectra. $^{28}$ With the increasing excitation wavelength, the emission maximum of the PDA nanoparticle solution shifts to the longer wavelengths (Figure $3 \mathrm{~b}$ ). Also, emission intensity is excitation wavelength dependent; it initially enhances with increasing wavelengths and then started to decrease gradually. The maximum emission intensities were recorded between the excitation wavelengths of 350 and $400 \mathrm{~nm}$. Therefore, we selected $370 \mathrm{~nm}$ as the excitation wavelength for DA sensing experiments.

Figure $3 \mathrm{c}$ shows the fluorescence spectra of $100 \mu \mathrm{M}$ DA solution recorded at different time intervals up to $6 \mathrm{~h}$. After $\mathrm{NaOH}$ addition, two emission peaks immediately formed at around 470 and $530 \mathrm{~nm}$, respectively. In the first $30 \mathrm{~min}$, intensities of the both peaks gradually increased. Interestingly, after $30 \mathrm{~min}$ the intensity of the peak around $530 \mathrm{~nm}$ started to decrease and it completely disappeared in $2 \mathrm{~h}$. The peak intensity around $470 \mathrm{~nm}$, on the other hand, decreased slightly between 30 and $60 \mathrm{~min}$ but after this point it started to increase again. After $6 \mathrm{~h}$, only an intense peak around $470 \mathrm{~nm}$ was (a)

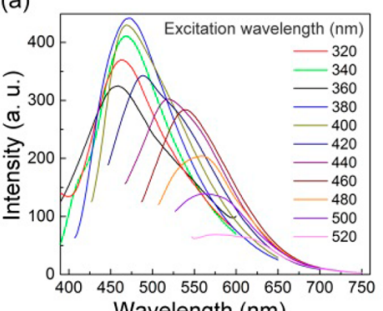

(b)

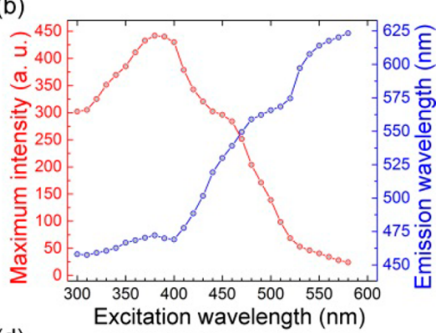

(c)

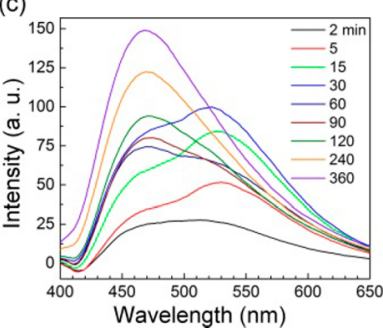

(d)

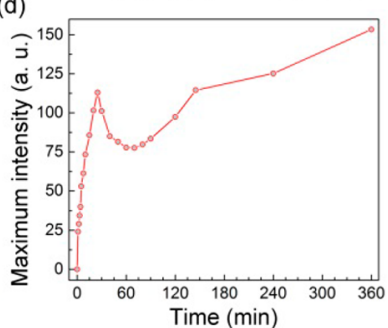

Figure 3. Emission properties of PDA nanoparticles: (a) excitation wavelength dependent fluorescence spectra of PDA nanoparticles, (b) change of the fluorescence intensity maxima and wavelength of PDA nanoparticle solution as a function of excitation wavelength, (c) time dependent fluorescence spectra of $100 \mu \mathrm{M}$ DA solution oxidized using $20 \mathrm{mM} \mathrm{NaOH}$, and (d) fluorescence intensity maximum change of the same solution over time.

observed. The peak around $530 \mathrm{~nm}$ may correspond to an intermediate product in the polymerization reaction, which is formed and consumed in $1 \mathrm{~h}$. We believe that in situ fluorescence measurements of the DA oxidation solution can give information about the structure of PDA, which is still not completely identified; ${ }^{31}$ however, it is beyond the scope of this study. Figure $3 \mathrm{~d}$ shows the emission intensity maxima of the same solution during polymerization. In the first minutes of polymerization, the fluorescence intensity sharply increased, after about $30 \mathrm{~min}$ it started to decrease, and after $90 \mathrm{~min}$ it began to raise again. Accordingly, we selected $30 \mathrm{~min}$ as the incubation time for sensing experiments.

The time dependent fluorescence spectrum of the DA oxidization solution can be a source of error in the analytical measurements. To overcome this problem and obtain stable emission characteristic over time, we added excess $\mathrm{HCl}$ to DA solutions after a certain time of oxidization and stopped the polymerization reaction by making the solution acidic. Figure 4a shows the fluorescence spectra of a DA oxidation solution $(100 \mu \mathrm{M})$ before and after $\mathrm{HCl}$ addition. In the first $15 \mathrm{~min}$, emission intensity increases gradually as expected. After $\mathrm{HCl}$ addition, the fluorescence spectrum of the solution shifted to shorter wavelengths and becomes very stable over time. In Figure $4 \mathrm{~b}$, the emission intensity at $510 \mathrm{~nm}$ was plotted as a function of time. After $\mathrm{HCl}$ addition intensity at $510 \mathrm{~nm}$ increased slightly and then it was almost constant for at least 45 min.

In order to evaluate the performance of the assay, we tested different concentrations of DA between 0.1 and $20 \mu \mathrm{M}$ with the assay. First, oxidation of dopamine solutions at different concentrations in PBS was initiated using $\mathrm{NaOH}(20 \mathrm{mM})$ and after $30 \mathrm{~min}$ excess $\mathrm{HCl}$ was added to stop the reaction. Then, fluorescence spectra of the DA solutions were recorded (Figure 5a). As expected with the increasing DA concentration, fluorescence of the solution becomes brighter. Figure $5 \mathrm{~b}$ shows the fluorescence intensities at $510 \mathrm{~nm}$ as a function of DA concentration. We observed that the response of the assay is 
(a)

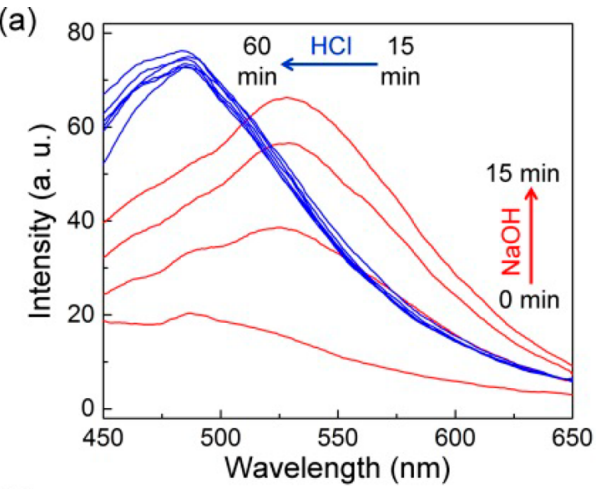

(b)

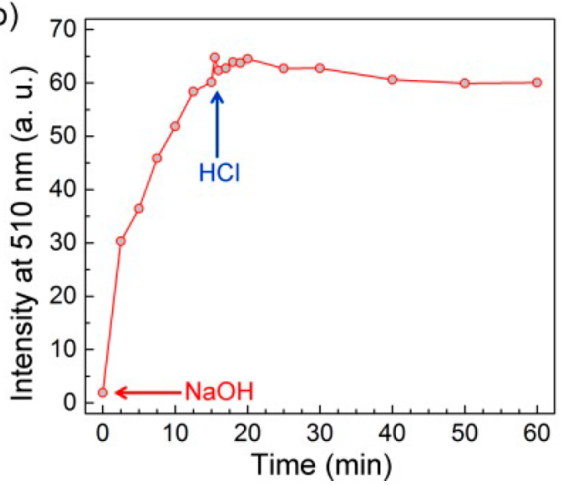

Figure 4. Stopping the polymerization reaction of DA using HCl: (a) fluorescence spectra of $100 \mu \mathrm{M} \mathrm{DA}$, which is oxidized using $20 \mathrm{mM}$ $\mathrm{NaOH}$ and after 15 min excess $\mathrm{HCl}$ was added; (b) fluorescence intensity at $510 \mathrm{~nm}$ change with time before and after $\mathrm{HCl}$ addition.

\section{(a)}

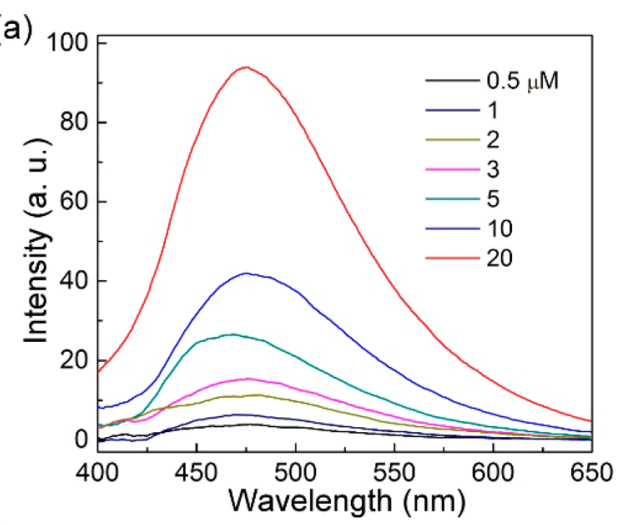

(b)

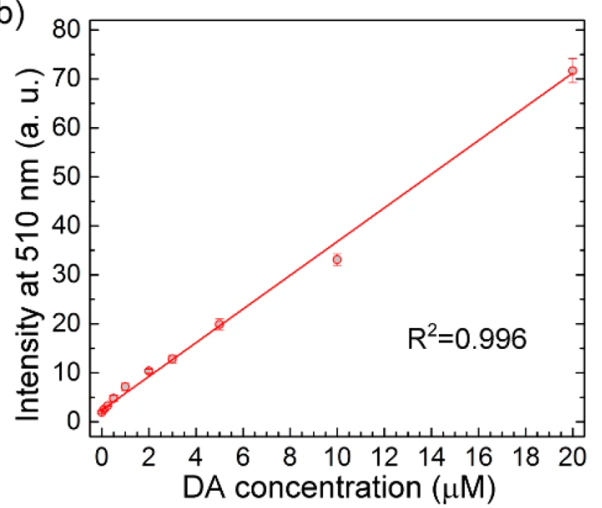

Figure 5. Sensitivity of the dopamine assay. (a) Fluorescence response of the assay against different concentrations of DA. (b) Assay response as a function of DA concentration which indicates the good linearity of the sensor in the studied region. highly linear $\left(R^{2}=0.996\right)$ in the studied concentration region. Relative standard deviations (RSD) of three separate experiments in Figure $5 \mathrm{~b}$ are between $0.2 \%$ and $2.4 \%$ indicating the good reproducibility of the sensor response. Using this calibration line, we calculated limit of detection (LOD) and limit of qualification (LOQ) values of the assay as $40 \mathrm{nM}$ and $120 \mathrm{nM}$, respectively. The achieved LOD and LOQ values of our fluorescence based assay is much better than absorption based methods (see the Supporting Information, Table S1) which typically have LOD and LOQ values above the miromolar level. In addition, to directly compare the performance of our fluorescence assay with the absorption based method, we measured the absorption of DA solutions which were oxidized using the same conditions with fluorescence experiments. We observed that for the concentrations above 1 $\mu \mathrm{M}$ there is a statistically significant difference in the absorption value between blank and DA solutions (see the Supporting Information, Figure S3). For the concentrations below $1 \mu \mathrm{M}$, the absorption assay could not detect the presence of DA, which is also in accordance with the previous reports. ${ }^{18-22}$

Lastly, we tested the selectivity of the assay using possible interfering chemicals such as AA, UA, amino acids, and sugars. The concentration of interfering substances is $100 \mu \mathrm{M}$. None of the tested chemicals produced a fluorescence signal even at this high concentration (Figure 6). To further demonstrate the

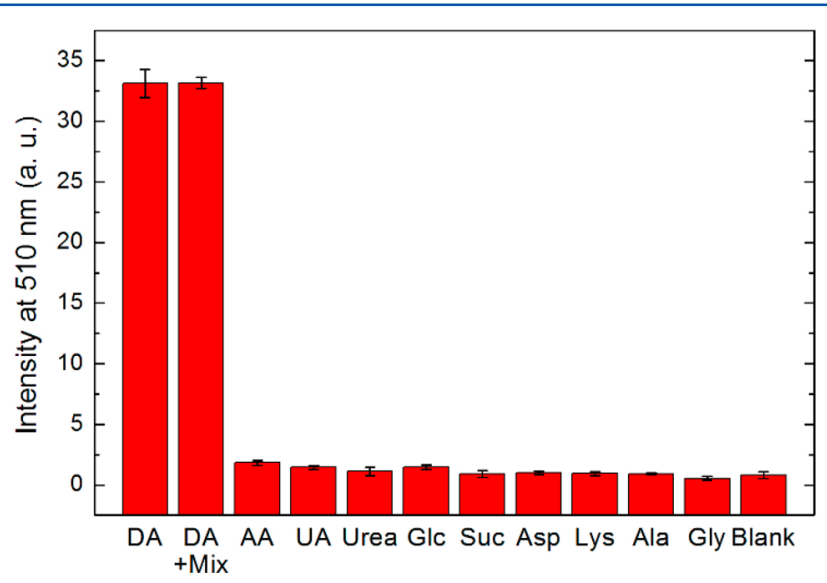

Figure 6. Selectivity of the dopamine assay. DA concentration is 10 $\mu \mathrm{M}$ and concentration of other substances is $100 \mu \mathrm{M}$. Fluorescence response was only observed for DA indicating the excellent selectivity of the assay (DA, dopamine; AA, ascorbic acid; UA, uric acid; Glc, glucose; Suc, sucrose; Asp, aspartic acid; Lys, lysine; Ala, alanine; Gly, glycine; Mix, mixture of all interfering chemicals).

selectivity of the method, we measured the fluorescence of 10 $\mu \mathrm{M}$ DA oxidization solution in the presence of mixture of all of interfering chemicals that used in this study (concentration of interfering chemicals was $100 \mu \mathrm{M}$ ). Presence of these chemicals in the DA assay has no effect on the fluorescence response (Figure 6). The observed excellent selectivity over DA of our assay is due to its specific response against PDA nanoparticle formation.

\section{CONCLUSION}

In conclusion, we developed a facile assay for detection of DA, which is based on measuring the fluorescence signal of the oxidation product of the DA. In order to determine optimum conditions for DA detection, we initiated the polymerization of $\mathrm{DA}$ at different oxidation conditions and recorded time 
dependent emission spectra of these solutions. Accordingly, we achieved a DA detection limit of as low as $40 \mathrm{nM}$. In addition, we did not observe any interference from AA and UA and as well as many amino acids and sugars. Compared with other DA detection methods, our assay is extremely simple and low cost, which make the method very promising for detection of DA deficiency related diseases.

\section{ASSOCIATED CONTENT}

\section{(S Supporting Information}

Table comparing performance of the assay with previous assays, time dependent UV-vis spectra of dopamine oxidation solution, in situ fluorescence spectra of dopamine solutions which were oxidized at different basic conditions, and a graph showing the sensitivity of the absorption based assay. This material is available free of charge via the Internet at http:// pubs.acs.org.

\section{AUTHOR INFORMATION}

\section{Corresponding Author}

*E-mail: bayindir@nano.org.tr. Phone: +90 312 2903500. Fax: +903122664365.

\section{Notes}

The authors declare no competing financial interest.

\section{ACKNOWLEDGMENTS}

We thank Fahri Emre Ozturk, Urandelger Tuvshindorj and Bihter Daglar for fruitful discussions. This work is supported by TÜBİTAK under Project No. 111T696. A.Y. is supported by a TÜBİTAK-BİDEB Ph.D. fellowship. M.B. acknowledges partial support from the Turkish Academy of Sciences (TUBA).

\section{REFERENCES}

(1) Lin, Y.; Chen, C.; Wang, C.; Pu, F.; Ren, J.; Qu, X. Chem. Commun. 2011, 47, 1181-1183.

(2) Ji, X.; Palui, G.; Avellini, T.; Na, H. B.; Yi, C.; Knappenberger, K. L.; Mattoussi, H. J. Am. Chem. Soc. 2012, 134, 6006-6017.

(3) Wightman, M. R.; May, L. J.; Michael, A. C. Anal. Chem. 1988, 60, 769A-779A.

(4) Li, B. R.; Hsieh, Y. J.; Chen, Y. X.; Chung, Y. T.; Pan, C. Y.; Chen, Y. T. J. Am. Chem. Soc. 2013, 135, 16034-16037.

(5) Tao, Y.; Lin, Y.; Ren, J.; Qu, X. Biosens. Bioelectron. 2013, 42, 4146.

(6) Nichkova, M.; Wynveen, P. M.; Marc, D. T.; Huisman, H.; Kellermann, G. H. J. Neurochem. 2013, 125, 724-735.

(7) Malem, F.; Mandler, D. Anal. Chem. 1993, 65, 37-41.

(8) Wu, W. C.; Chang, H. W.; Tsai, Y. C. Chem. Commun. 2011, 47, 6458-6460.

(9) Qian, T.; Wu, S.; Shen, J. Chem. Commun. 2013, 49, 4610-4612. (10) Wang, H.; Jin, H.; Zhang, H. S. Fresenius J. Anal. Chem. 1999, 365, 682-684.

(11) Peterson, Z. D.; Collins, D. C.; Bowerbank, C. R.; Lee, M. L.; Graves, S. W. J. Chromatogr., B 2002, 776, 221-229.

(12) Mao, Y.; Bao, Y.; Han, D. X.; Li, F. H.; Niu, L. Biosens. Bioelectron. 2012, 38, 55-60.

(13) Qu, K.; Wang, J.; Ren, J.; Qu, X. Chem.-Eur. J. 2013, 19, $7243-7249$.

(14) Chen, J. L.; Yan, X. P.; Meng, K.; Wang, S. F. Anal. Chem. 2011, 83, 8787-8793.

(15) Xu, Q.; Yoon, J. Chem. Commun. 2011, 47, 12497-12499.

(16) Mu, Q.; Xu, H.; Li, Y.; Ma, S.; Zhong, X. Analyst 2014, 139, 9398.

(17) Baron, R.; Zayats, M.; Willner, I. Anal. Chem. 2005, 77, 15661571.
(18) Nevado, J. J. B.; Gallego, J. M. L.; Lauguna, P. B. J. Pharm. Biomed. Anal. 1996, 14, 571-577.

(19) Berzas, J. J.; Lemus, J. M.; Buitrago, P. Anal. Lett. 1997, 30, $1109-1120$.

(20) Da Cruz Vieira, I.; Fatibello-Filho, O. Talanta 1998, 46, 559564.

(21) Abbaspour, A.; Khajehzadeh, A.; Ghaffarinejad, A. Analyst 2009, 134, 1692-1698.

(22) Abbaspour, A.; Valizadeh, H.; Khajehzadeh, A. Anal. Methods 2011, 3, 1405-1409.

(23) Lee, H.; Dellatore, S. M.; Miller, W. M.; Messersmith, P. B. Science 2007, 318, 426-430.

(24) Wei, Q.; Zhang, F.; Li, J.; Li, B.; Zhao, C. Polym. Chem. 2010, 1, $1430-1433$

(25) Hong, S.; Na, Y. S.; Choi, S.; Song, I. T.; Kim, W. Y.; Lee, H. Adv. Funct. Mater. 2012, 22, 4711-4717.

(26) Della Vecchia, N. F.; Avolio, R; Alfe, M.; Errico, M. E.; Napolitano, A.; d'Ischia, M. Adv. Funct. Mater. 2013, 23, 1331-1340.

(27) Olsen, S.; Riesz, J.; Mahadevan, I.; Coutts, A.; Bothma, J. P.; Powell, B. J.; McKenzie, R. H.; Smith, S. C.; Meredith, P. J. Am. Chem. Soc. 2007, 129, 6672-6673.

(28) Nighswander-Rempel, S.; Riesz, J.; Gilmore, J.; Meredith, P. J. Chem. Phys. 2005, 123, 194901-194906.

(29) Meredith, P.; Powell, B. J.; Riesz, J.; Nighswander-Rempel, S. P.; Pederson, M. R.; Moore, E. G. Soft Matter 2006, 2, 37-44.

(30) Zhang, X.; Wang, S.; Xu, L.; Ji, Y.; Feng, L.; Tao, L.; Li, S.; Wei, Y. Nanoscale 2012, 4, 5581-5584.

(31) Dreyer, D. R.; Miller, D. J.; Freeman, B. D.; Paul, D. R.; Bielawski, C. W. Langmuir 2012, 28, 6428-6435. 